\title{
La Protección de las Inversiones Extranjeras
}

\author{
por el Dr. MANUEL R. GARCIA-MORA *
}

La iniciación de un nuévo régimen, la inauguración de una nueva política económica y el surgimiento de un nuevo Estado en la Comunidad Internacional van frecuentemente acompañados o seguidos por una nacionalización en gran escala de las inversiones extranjeras. Esta politica parece ser el producto de la ola de nacionalismo que precede generalmente los cambios revolucionarios. De hecho, este fenómeno del nacionalismo ${ }^{1}$ se ha producido en forma particularmente frecuente desde los años de la postguerra, cuando una revo'ución social de gran magnitud se extendió de un pais a otro en lo que parece ser una inevitable sucesión. Lo atestiguan las nacionalizaciones en Irán, Egipto, Indonesia, Guatemala, y más recientemente en Cuba, para no mencionar las que ocurrieron mucho antes en Méjico, la Unión Soviética, la Europa Occidental y los paises de la Europa Central. Desde que no puede haber ninguna duda de que, por una parte, existe un derecho internacional de propiedad ${ }^{2} y$, por otra, que un Estado tiene la facultad de expropiar la propiedad para uso público, se plantea inicialmen. te la cuestión vital de como conciliar estos dos principios aparentemente contradictorios, de manera que las inversiones de capitales puedan ser debidamente protegidas. Este problema no afecta solamente las buenas relaciones entre las naisones, sinó también la paz y la seguridad de la humanidad. Por consiguiente, este trabajo tiene por objeto hacer resaltar los métodos por medio de los cuales podrá asegurarse en la mejor y más adecuada forma posible la seguridad de las inversiones.

- Profesor de Derecho, Fordham University, New York, N.Y.

I En el aspecto teórico, se hace una distinción entre "nacionalización" y "exprom piación". La primera designa un secuestro general de la propiedad, mientras que la segunda se aplica generalmente al secuestro especifico de determinada industria, negocio o empresa. Ver Domke, "Foreign Nationalization: Some Aspects of Contemporary International law", 55 Am. J. Int. L. 585, 588 (1961).

2 Ver al respecto el Articulo 17 de la Declaración Universal de Derechos Humanos, adoptada por la Asamblea General de las Naciones Unidas en 1948, que dice que "todos tienen el derecho de poseer propiedad en particuiar o asociados con otros" y que "Nadie podrá ser arbitrariamente privado de su propiedad". 


\section{Los principios del Derecho Internacional tradicional}

En su más sencillo término, "nacionalización" es apoderarse de la propiedad privada para uso público. Sin embargo, la experiencia demuestra que un Gobierno puede privar a una persona ciel uso de su propiedad de distintas maneras. Por ejemplo, la arbitraria prohibición de exportar bienes; tasas exhorbitantes como las impuestas por la Ley Minera Cubana, del 27 de Octubre de 1959: la designación de interventores especialmente cuando los dueños están ausentes, como se ha sugerido recientemente en el Congo contra intereses comerciales Belgas ${ }^{3}$; violación de concesiones, y así por el estilo, son todos medios de control efectivo del uso de la propiedad privada. Es por esta razón que, con fines de protección, debe adoptarse un amplio concepto de nacionalización. Con este objeto, la Convención de Harvard sobre la Responsabilidad Internacional de los Estados por Daños a los Extranjeros, define "secuestro" de la siguiente manera:

"Un "secuestro" incluye no so'areente una apropiación ilegal de la propizdad sinó también toda interferencia no razonable con el goce o disposición de la propiedad, que justifique una situación que impida que el dueño pueda usarla, gozarla o disponer de la propiedad dentro de un periodo de tiempo razonable después de acreditada tal interferencia"4.

Por consiguiente, es claro que cualquier acto de parte del Gobierno que prive al dueño del uso, goce o disposición de su propiedad puede ser legalmente considerado como un secuestro que compromete su responsabilidad internacional.

Quienes están familiarizados con el derecho internacional recordarán que bajo el antiguo derecho era sencillo para un gobierno emplear la fuerza contra otro Estado para proteger las inversiones de sus ciudadanos, bajo un alegado derecho de propia-defensa ${ }^{5}$. Sin embargo, de acuerdo con el derecho internacional contemporáneo, tales acios son legalmente inadmisibles, y un Gobierno, que actuase de esta manera podría considerarse como realizando un acto de agresión ${ }^{8}$. Con respecto a esto debería puntualizarse que constituye un interés vital para la Comunidad Mundial establecer la reg'a de derecho en las relaciones entre naciones y este postulado exige imperativamente que los Gobiernos acudan a medios pacíficos para resolver sus litigios internacionales?.

s Domke, nota it supra I, p 589.

- Artículo 10, par. 3 (a). Para el texto de este Convenio, ver Proc. Am. Soc. Int. Law. 102 (1960).

B Thomas $\&$ Thomas. Non-Intervention: The Law and its Import in the Americas. c. XV (1956).

- La intervención de Gran Bretaña y Francia en Egipto, con motivo de la Nacionalización por Egipto da la Cia. del Canal de Suez es un caso sobre este punto.

1 Bishop. "The International Rule of Law", 59 Mich. L. Rev. 553 (1961). 
Podria agregarse que el Estado agraviado no queda del todo sin defensa, ya que pueden hacerse valer principios bién establecidos, principios que convierten en ilegal una nacionalización bajo ciertas condiciones. Es así como, un antiguo principio del derecho internacional establece que los Estados están especificamente obligados a respetar los derechos adquiridos de los extranjeros, y que cuando la legislación que afecta estos derechos es contraria a la politica general de Estados civilizados y cuando infringe el principio de la igualdad jurídica, se considera un acto injusto que compromete la responsabilidad del Estado. Este principio sugiere claramente la posibilidad que, bajo ciertas condiciones, la nacionalización o expropiación de la propiedad puede ser, en sí, contraria al derecho internacional y, por consiguiente, un acto tortuoso que compromete la responsabilidad del Estado. Esta proposición puede ilustrarse mediante el conocido caso Chorzow-Factory, resuelto por la Corte Permanente de Justicia, en 1927, cuando la Corte estableció esencialmente que la nacionalización que es violatoria de las cláusulas de un tratado no constituye una expropiación, sinó el "secuestro" de la propiedad, derechos e intereses que no podrian expropiarse aún mediante la compensación", salvo ciertas condiciones establecidas en el tratado. ${ }^{8}$ Esta decisión es, ciertamente, de importancia capital en el mundo contemporáneo, pues podrá verse subsecuentemente que la única protección real para la propiedad extranjera es la que está prevista mediante convenciones bilaterales o multilaterales.

En segundo lugar, se podría argumentar fundadamente que la propiedad privaria. Es así como el artículo $24(1)$ de la Constitución Cubana de recurre a ella es invalidada bajo el punto de vista del derecho internacional. Podría ser sorprendente saber que la constitución de muchos países que proceden a nacionalizaciones garantizan el derecho de tener y usar la propiedad privada. Es asi, como el artículo $24(1)$ de la Constitución Cubana de 1940 prohibe la confiscación de la propiedad, y el artículo 87 de la Ley Fundamental de Cuba del 7 de Julio de 1959, respeta la "existencia y legitimidad de la propiedad privada en su más amplio sentido como función social..." 9 . De la misma manera, el articulo 6 de la Constitución Iránia de $1907^{10}$, y Jos artículos 9 y 10 de la Constitución Egipcia de $1923^{11}$, para sólo mencionar paises donde se ha procedido recientemente a nacionalizaciones, todas garantizan la propiedad privada, tanto a sus ciudadanos. como a los extranjeros ${ }^{12}$. El significado de estas disposiciones consiste en que el secuestro arbitrario de la propiedad privada en dichos paises corre el riesgo de ser

8 Chorzow Pactory Case, P.C.I.J., Ser. A., N॰ 9, pp. 27, 31 (1927).

- Caso mencionado por Domke, nota ut supra 1, p. 587.

102 Peaslee, Constitutions of Nations, 206 (1950).

11 Ibid. Vol, 1, p. 721.

12 Para una discusión sobre las Constituciones y Reglamentaciones de algunos paises del Extremo Oriente, ver Alexander. "Foreign Investment Laws and Regulations of the Countries of Asia and the Far Fast", 1 Int' 1 \& Comp. L. Q. (1952). 
contrario a sus propias constituciones, $y$, por consiguiente, al derecho internacional. Un autorizado planteamiento de esta posición se encuentra en el reclamo de la Norwegian Shipping contra los Estados Unidos, resuelto en 1922 por la. Corte Permanente de Arbitraje, y que se refería a reclamos planteados contra los Estados Unidos por súbditos noruegos, cuyos contratos para la construcción de barcos habian sido asumidos por los Estados Unidos durante la Primera Guerra Mundial ${ }^{13}$. La Corte otorgó una compensación a los súbditos noruegos, sobre la base inter alia, que los Estados Unidos estaban en la obligación de resfetar los derechos de propiedad de los súbditos extranjeros o de pagar una justa compensación a los reclamantes, "tanto de acuerdo con la Ley Municipal de los Estados Unidos, como con el Derecho Internacional, basados en el respeto a la propiedad privada". Conviene mencionar que la Corte hizo una referencia a la quinta Enmienda a la Constitución de los Estados Unidos, según la cual ninguna persona podrá ser privada de la vida, la libertad o la propiedad sin previo juicio de derecho, y agregó que "Es de general zonsenso que, a este respecto, el derecho público de las Partes se halla en completo ácuerdo con el derecho público internacional de todos los países civilizados" ${ }^{14}$. Lo que insinuaba la Corte era sencillamente que el respeto de la propiedad privada constituye un principio general del derecho reconocido por las naciones civilizadas, que el artículo 38 (lc) de los Estatulos de la Corte Internacional de Justicia considera hoy como una fuente del Derecho Internacional. Esto, como se comprende, constituye un concepto clave de la protección internacional, pues indica claramente que, desde que la mayor parte de las naciones tienen disposiciones constitucionales que respetan la propiedad privada, su arbitraria violación constituye un delito internacional. La conclusión es, por consiguiente, indudable, y es que, según el derecho internacional, existe un derecho de protección, y se ha visto que este derecho ya ha sido défendido en la práctica, en innumerables casos, y, particularmente, para el cumplimiento de obligaciones y contratos internacionales. También debe quedar en claro que estos constituyen argumentos legales que pueden hacerse valer ante una Corte de Justicia.

\section{II.-- Asuntos en los que se puede hacer valer la protección diplomática}

El método más usual de protección a las inversiones es el de la protec. ción diplomática, la que en este aspecto, es el uso de las vias diplomáticas para asegurar que las inversiones no sean arbitrariamente apropiadas. Pero la protección diplomática se menciona en los términos de un dilema. Se ha visto, por una parte, que el derecho internacional sugiere un derecho inter. -nacional de propiedad que estaria protegido por el derecho internacional.

13 Para el texto que se refiere a esta opinión, ver 1 . Schwarzenberger, International Law as Applied by International Courts and Tribunals, 100-101 (2a. ed. 1949).

14 Ibid., p. 101. 
Pero al mismo tiempo existe el derecho indiscutible de un pais para estable cer programas económicos y sociales en favor de sus propios nacionales y para adoptar las medidas necesarias para realizarlos. Está incluidã en éstas el derecho del gobierno para reclamar la posesión de la propıedad privada de manera que se ha'le efectivamente intzgrada a la vida económica del pais. Debería recordarse, al respecto, que, aún con relación a los nacionales, sus derechos de propiedad se modifican constantemente ${ }^{15}$. Es precisamente por este motivo que el derecho de nacionalizar la propiedad privada no es discutido seriamente por ningún gobierno ${ }^{16}$. Podria aún sugerirse que el derecho internacional garantizara tal derecho. La evidencia de los hechos indica que las nacionalizaciones han sido más frecuentemente discutidas deb:do a la forma como se han llevado a cabo. Por consiguiente, según principios aceptados, la intervención diplomática es fermitida a un Estado bajo dos condiciones: 1) si las inversiones de sus naciona'es son nacienalizadas en forma discriminatoria; y 2) si una justa y pronta compensación no es pagada a sus dueños. Debería quedar en claro, por la mera enunciación de estas condiciones, que la protección diplomática, no está realmente encaminada a evitar la nacionalización. Su finalidad es principalmente la de asegurar que, si las inversiones han sido secuestradas, el dueño no será arbitrariamente privado de su valor. Como estas dos condiciones son las únicas sobre las que puede ejercerse la intervención gubernamental, es altamente conveniente discutir'as con algún detalle.

\section{Discriminación.}

Los decretos de nacionalización han sido usualmente aplicados en forma discriminatoria contra los intereses de los nacionales en determinados países. No es un secreto que las inversiones de los ciudadanos Norte-Americanos. casi exclusivamente, son los que han sido, hoy dia, nacionalizados en Cuba. Deberia comprenderse claramente, sin embargo, que toda nacionalización que últimamente ha recaido sobre intereses extranjeros tiene cier:o aspecto discriminatorio. Es asi como la nacionalización del petróleo en Méjico, allá por los años de 1930, recayó exclusivamente sobre intereses Norte-Ameri-

15 Es de general conocimiento que Gran Eretafia inició un extenso programa de nacionalización después de la Segunda Guerra Mundial. Lo mismo hicieron Francia e Italia. Esto es significativo, pues, en lo que se refiere a Gran Bretaffa y Francia, una larga tradiçón de constitucionaïismo caracterizó su desarrollo politico. Por consiguiente, la nacionalización no es un monopolio de los gobiernos totalitarios. Ver Doman. "Postwar Nationalization of Foreign Prcperty in Europe". 48, Colum. L. R. 1125 (1948).

16 Es asi como, con respecto a la nacionalización iniciada por el Gobierno Meiicano, el Gobierno de los Estados Unidos no discutió el derecho de Méxíco para esa nacionalización. Las medidas de nacionalización fueron discutidas porque negabin arbitrariamente una justa compensación a los ciudadanos Americanos. Para la relación de las comunicaciones entre Estados Unidos y México, ver 3 Hackwort, Digest of International Law. 655-665 (1942). 
canos. Británicos y Holandeses, desde que ningún Mejicano o ciudadano de otro pais poseía entonces petróleo. El tipo de nacionalización que es discriminatorio y. por consiguiente, ilegal, es el que va dirigido a la nacionalidad del dueño y nó a la naturaleza de la propiedad. Esta proposición puede ser mejor ilustrada por la nacionalización Indonesa de 1958, que estaba especificamente y exclusivamente dirigida contra "las empresas de propiedad holandesa que se hallaban dentro del territorio de la república de Indonesia" 1 . Más recientemente, la Ley Cubana de Nacionalización, No 851, del 6 de Julio de 1960, estuvo exclusivamente dirigida contra los intereses de propiedad Norte-Americana. El carácter discriminatorio de esta Ley apai ece en el articulo $1^{\circ}$ que dice que:

“El Presidente y el Primer Ministro están autorizados para ordenar... la nacionalización, mediante expropiación, de las propiedades o bienes pertenecientes a personas juridicas o nacionales de los Estados Unidos de América o de los negocios, en los cuales dichas personas tuvieran interés o participación mayoritaria, aún si estuvieran organizados de acuerdo con las leyes de Cuba" ${ }^{18}$.

No puede dudarse de que esta discriminación está ampliamente motivada por la hostilidad politica y por sentimientos anti-extranjeros de parte del gobierno para lograr cierta popularidad interna. Esta actitud explica parcialmente porque las medidas de nacionalización se anuncian en términos politicos de orden más elevado, generalmente relacionados con la liberación o la independencia del respectivo pueblo. Es asi como se puede notar que la nacionalización de los intereses de propiedad holandesa en Indonesia fue oficialmente justificada, por lo menos en el orden interno, como una medida adicional "dentro del marco de la lucha para la liberación del Irian Brast (Nueva Guinea Occidental), contra las empresas de propiedad holandesa que se hailaban en el territorio de la República de Indonesia" 19. Y el Gobierno Cubano ha pretendido que sus medidas de nacionalización tienen por fina'idad proteger a Cuba contra la "agresión económica americana" ${ }^{20}$. Estos pronunciamientos constituyen evidencia adicional del sentido discriminatorio de las nacionalizaciones en Indoresia y Cuba.

Teniendo en cuenta los hechos anteriores, debe constituir un principio bien establecido que la discriminación contra los extranjeros en la forma señalada, constituye una violación del derecho internacional. La razón de ser de este principic es que, por una razón que no se justifica, los extranjeros son tratados en forma distinta de los nacionales, y aún si los extranjeros y los nacionales son a veces tratados de igual manera. siempre se presenta la objeción de que los extranjeros están obligados a financiar un programa

17 Texto tal como figura en Domke, nota ut supra 1, p. 698, n. 92.

18 Fara el texto de esta ley, ver 55 Am. J. Int. L. 822 (1961).

19 Como $\mathrm{f}_{\mathrm{i}}$ ura en Domke. "Indonesian Nationalization Measuras before Foreign Courts", 54 Am. J. Int. L. 305 (1960).

20 Para el texto, ver 43 Dept. State Bull. 401 (1960). 
social y económico del cual no obtienen ningún beneficio. No es preciso hacer un gran esfuerzo para percibir la injusticia que representa esta politica, porque no es de ninguna manera imposible que los programas de mejoramiento social no sean, a fin de cuentas, llevados a cabo a expensas de los intereses extranjeros.

La naturaleza esencialmente discriminatoria de la reciente nacionalización Cubana ha sido considerada por una Corte Distrital de los Estados Unidos en el asunto: Banco Nacional v. Sabbatino, ${ }^{21}$, en el que la Corte, de manera inequivoca, ha declarado que:

“. . la presente forma de nacionalización es contraria a los principios del derecho internacional, por su naturaleza discriminatoria. Ei acto realizado clasifica a los nacionales de los Estados Unnidos separándolos de todos los demás y no establece ninguna base razonable para tal clasificación. El decreto no justifica la clasificación sobre la base de la conducta de los dueños en la dirección y explotación de sus propiedades teniendc en cuenta su importancia para la seguridad del Estado donde reside el dueño de la propiedad. La justificación constituye sencillamente una represalia contra otro gobierno. Sin duda que las medidas que los Estados pueden emplear en sus rivalidades son de gran variedad, pero no incluyen, la confiscación de la propiedad de los nacionales del gobierno rival" 22 .

El anterior es un principio bien establecido, afirmado muchas veces por los gobiernos, las Cortes, y los tratadistas que se han ocupado de estos asuntos. Es de cierta significación, agregar, que, en reciente estudio sobre esta materia, el Doctor Martin Domke reunió un número apreciable de opiniones autorizadas para demostrar que la nacionalización discriminatoria es considerada ilegal, aún por los juristas de países comunistas" ${ }^{23}$.

\section{Pago por compensación.}

La falta de pago de una justa y pronta compensación por la propiedad expropiada es el segundo aspecto importante sobre el que se puede solicitar la protección diplomática. Sin duda, la falta de compensación invalida una medida de nacionalización y la asemeja a una confiscación. Este asunto fue bién puesto en evidencia por el Secretario de Estado en 1938, con motivo de la nacionalización del petróleo por el Gobierno Mejicano. El Secretario de Estado declaró entonces con firmeza que "El aprovecharse de la propiedad sin compensación no constituye expropiación. Es confiscación. No deja de ser confiscación, aunque exista un intento manifestado de pagar alguna vez

21193 F. Supp 375 (S.D.N.Y.) - (1961).

22 Ibid. p. 385.

23 Domke, "Foreign Nationalization: Some Aspẹcts of Contemporary International Law". 55. A. J. Int, Law, 585, 603 (1961). 
en el futuro" 24. El pago de la compensación es, por consiguiente, generalmente aceptado como un requisito previo de la nacionalización, y es de algún interés anotar que las recientes leyes de nacionalización, de Irán, Egipto, Indonesia y Cuba, contienen disposiciones sobre compensación. Pero esto, en sí, no es suficiente, pues la experiencia demuestra que frecuentemente los Gor biernos se han apoderado de la propiedad sin compensación, a pesar de las disposiciones en este sentido, basándose en razones tales como su imposibilidad de pagar, la importancia social del motivo por el cual se ha apoderado. de la propiedad, y a veces el pago es diferido o se ofrece un pago parcial. Desde luego, es evidente que una compensación en estas condiciones no constituye compensación alguna. Este asunto fue promovido por el Secre. tario de Estado, en 1953, en un litigio con Guatemala sobre la expropiación de ciertas proniedades agricolas de la "United Fruit Company". El Secretario de Estado dijo, en aquella ocasión, que "Justa compensación puede definirse como la compensación que ...es pronta, es adecuada y es efectiva, de lo contrario la compensación no es justa" ${ }^{25}$. Más recientemente, la Ley Cubana de Nacionalización, del 6 de Julirs de 1960, dispuso aue la compensación sea pagada en bonos, con plazos de amortización no menores de treinta años, con intereses no menores del dos por ciento, que serán pagados de un Fondo constituido anualmente con el $25 \%$ del valor del comercio exterior que corresponda a la mayor adquisición de azúcar efectuada cada año por los Estados Unidos sobre la base de tres millones de toneladas ${ }^{26}$. Desde que el referido Fondo se estableció a base da las compras de azúcar por los Estados Unidos, la supresión por éstos de la cuota de importación de azúcar cubana ha convertido el pago de la compensación en algo teórico, para no decir más ${ }^{27}$.

Una Corte Distrital de los Estados Unidos tomó nota de esta situación al ocuparse del asunto del Banco Nacional de Cuba v. Sabbatino", que ya hemos mencionado, l'egando a la conclusión que:

"La condición establecida para el pago de los intereses de los bonos, asi como la inseguridad del pago a su término, hace que los bonos no puedan cotizarse en el mercado (bolsa) y que no tengan valor. Aún más, el valor de la propiedad expropiada debe ser únicamente fijado por péritos designados por el Gobierno Cubano, un adversario conocido de los intereses de las personas cuyas propiedades han sido capturadas. Claramente, esto no constituye adecuada compensación dentro de las normas del derecho internacional" 29 .

24 Nota del Secretario de Estado Hull al Embajador Mejicano en Washington, 21 de Fulio de 1938, 3 Hackworth, op. cit., nota ut supra 16, p. 656.

25 Bull. Dept. State 29, p. 357 (1953).

28 Para el texto de esta ley, ver 55 Am. J. Int. Law, 822 (1961).

27 Ver Ley P. 86-592, Julio 6 de 1960, 74 Stat, 330, 7 U.S.C. 1158.

28193 F. Supp. 375 (S.D.N.Y. 1961).

29 Id., p. 386. 
El principio de la justa compensación ha sido sostenido en forma semejante por tribunales internacionales. Es de indiscutible autoridad al respecto el asunto de la "Norwegian Shipping Company" contra los Estados Unidos, que se referia a contratos para la construcción de buques, que fueron asumidos por el Gobierno de los Estados Unidos. Un Tribunal arbitral estableció que la finalidad de la justa compensación es una "completa restituciốn del status quo ante, basado no en las futuras ganancias de los Estados Unidos u otras potencias, sinó sobre las bases de las ganancias y pérdidas de los propietarios noruegos, comparados con los de otros dueños de propiedades similares" ${ }^{30}$. Se podría mencionar otros casos en los que se ha llegado a conclusiones similares ${ }^{31}$.

Se podria agregar que, en politica internacional, hay casos en los que el principio de la justa compensación ha dado lugar a consideraciones tales como la inestabilidad del pais nacionalizado o su incapacidad bona fide para pagar. En tales casos, el Gobierno de los Estados Unidos ha reconocido esos hechos, haciendo, por consiguiente, del pago de la justa compensación una condición bastante flexible. Es asi ccmo, en la solución de los reclamos de los ciudadanos Norte-Americanos contra Yugoeslavia, por acuerdo del 19 de Julio de 1948, el Gobierno de los Estados Unidos se mostró dispuesto a aceptar la suma de diecisiete millones de dólares para el pago de la propiedad nacionalizada, aún cuando era evıdente que el valor de la propiedad en el mercado era muy superior ${ }^{32}$. De la misma manera, por el Convenio sobre Reclamos, del 19 de Noviembre de 1941, Méjico convino en abonar a los Estados Unidos cuarenta millones de dólares por el valor de la propiedad expropiada, lo que representaba para los ciudadanos Norte-Americanos interesados una pérdida de 23.995 .991 dólares ${ }^{33}$.

La reg'a que establece el pago de compensación es justa desde otro punto de vista. Mientras se admite generalmente que un Gobierno tiene el derecho - -aún el deber- de fomentar el desarrollo social y conómico de sus naciona'es es, por lo mismo, cierto que tales programas ino pueden ser llevados a cabo a expensas de los ciudadanos de otro país ${ }^{34}$. De aqui que la regla que exige compensación no es solamente la expresión de una rrgla de justicia universalmente reconocida; es también esencial para el bienestar de la comunidad mundial, pues sin ella ningún negocio comercial o financiero

so 1 U.N. Rep. of Arb. Awards, 309, 333 (1948).

31 Para estos casos, ver Bishop. International Law; Cases and Materials, 485-487 (1953).

32 Kuhn, "Natonalization of Foreian Ownes Property in its Impact on Irternational Law", 45 Am. J. Int. L. 709. 710 (1951).

s3 Tal como informa Carlston. "Concession Agreement and Nationalization", 52, Am. J. Int. L. 260,273 (1958).

34 Nota del Secretario de Estado Hull al Embajador de México en Washington, 3 Hackworth, op. cit. nota ut supra 16, p. 657. 
podria llevarse a cabo en el orden internacional con seguridades para las naciones ${ }^{35}$.

\section{Protección por medio de Convenios Multilaterales y Bilaterales}

Los métodos señalados en las páginas anteriores están verdaderamente destinados a obtener la entrega a los inversionistas del monto de sus inversiones, en el caso de producirse la nacionalización. Sin embargo, bajo las actua'es circunstancias con el incremento de las necesidades de los paises sub-desarrollados para lograr inversiones de capitales, el problema de atraer capitales y ofreceries al mismo tiempo alguna protección, ha adquirido más importancia, quiénes están designados para proteger a los inversionistas contra la nacionalización, se han preocupado, por consiguiente, en primer lugar, de adoptar medidas que tengan por objeto asegurar el pago de las compensaciones. El Gobierno de los Estados Unidos ha favorecido la negociación de Tratados Multilaterales y Bilaterales para lograr esta finalidad. La primera tentativa en este sentido se efectuó en Bogotá, en 1948, cuando se firmó un convenio entre las repúblicas Americanas para la protección de las inversiones extranjeras, que dispone lo siguiente:

"Los Estados no adoptarán acción discriminatoria contra inversiones por medio de la cual las empresas de capital extranjero puedan ser privadas de los derechos de propiedad adquirida legalmente, por razones o bajo condiciones diferentes de las que la Constitucićn o leyes de cada país señalan para la expropiación de la propiedad de los nacionales.

Toda expropiación deberá estar acompañada del paģo de un precio conveniente de manera pronta, adecuada y efectiva ${ }^{36}$ ".

Sin embargo, fueron tantas las reservas formuladas a esta disposición que la Convención no logró entrar en vigencia. El Gobierno de los Estados Unidos inició entonces un programa para la firma de tratados bilaterales con el mismo propósito. Es particularmente instructivo al respecto el Tratado de Amistad, Comercio y Desarrol'o Económico, firmado el 23 de Ncviembre de 1949, entre los Estados Unidos y el Uruguay. El artículo 8 se refiere a la protección reciproca de la propiedad de los ciudadanos de eștos países en la siguiente forma:

"La propiedad de los nacionales o compañias de cualesquiera de las Partes deberá recibir la más constante protección y seguridad dentro del territorio de la otra Parte...

35 Becker, "Just Compensation in Expropiation Cases; Decllne and Partial Recovery", Proc. Am. Soc. Int. L. 336, 344 (1959). Este articulo ha sido publicado en la Revista "Derecho", № XIX, págs. 19 a 28. (Nota Ed.).

36 Articulo 25. - Como mencionado en un discurso del Hon. James G. Fulton, ante la Sociedad Americana de Derecho Internacional. Ver. Proc. Am. Soc. Int. L. 200, 203. (1958)

37 Para el texto, ver 23 Dept. State Bull. 502 (1950). 
Cualquicr expropiación deberá hacerse de acuerdo con las leyes vigentes, las que deberán por lo menos asegurar el pago de una justa compensación de manera pronta, adecuada y efectiva".

Una rápida lectura de esta disposición revela que no es realmente una cláusula de verdadera protección para los inversionistas, pues los requisitos de la compensación están señalados en los términos de "pronta", "adecuada" y "efectiva", que anteriormente han probado sér de dudoso valor. Tal vez es de mayor importancia la claúsula que hace depender enteramente el pago de la compensación de la ley locai.

Bajo este aspecto, es muy dudoso que una verdadera protección se haya efectivamente logrado. De igual manera, el Tratado de Amistad, Comercio y Navegación, firmado el 2 de Abril de $1953^{39}$ entre los Estados Unidos y e! Japón, contiene una claúsula protectora de las inversiones, bastante parecida a la anterior disposición, en su artículo VI, inciso 1; además, el inciso 3 del mismo artículo indica ia manera como deberá electuarse el pago de la compensación. Dice asi:

"La propiedad de los nacionales y las Compañias de cualesquiera de las Partes no podrá ser apropiada dentro del territorio de la otra Parte, salvo para un interés público, y no podrá serlo sin el pronto pago de una compensación. Esta compensación deberá efectuarse en forma efectivamente realizable y deberá representar el equivalerite total de la propiedad expropiada; y una provisión (depósito) deberá haberse efectuado antes o en el instante de la apropiación para la fijación y el pago consiguiente".

La fuerza de esta disposición se halla muy disminuida por el articulo XII, inciso 3. que, al referirse a una transferencia razonable de compensacién, agrega que cstá sujeta a "iodo control de cambios que sea necesario para asegurar la importación de mercaderías y servicios esenciales para el bienestar del pueblo japonés" " . Idénticas disposiciones se ha!lan en el Tratado de Amistad, Comercio y Navegarión, firmado, el 24 de Octubre de 1954, entre los Estados Unidos y Alcmenia Occidental ${ }^{41}$, y en el más reciente Tratado del 3 de Abril de 1961, entre los Estados Unidos y la República del Viet-Nam ${ }^{42}$. La dificultad fundamental consiste en que en ninguno de estos Tratados se dispone sobre el pago no calificado de compensación en el caso de nacionalización, y es, por esta razón que uno queda con la impresión que no logran ofrecer adecuada protección a los inversionistas.

38 Subrayado por el autor.

s9 Para el texto, ver 4 U.S.T. \& O.I.A. 205.

40 Tal como figura en Gardner, "International Measures for the Promotion and Protection of Foreign Investment". Proc. A. Soc. Int. L. 255, 260 (1959).

41 Articulo V. par. 4., Para el texto ver 7 U.S.T., 1839

42 Para el texto, ver 44 Dept. State Bull. 652 (1961). 
Por otra parte, un conjunto de tratados establece de manera precisa un sistema para obtener moneda extranjera para el pago de compensaciones. Fsto puede ser mejor demostrado por el Tratado de Amistad, Comercio y Navegación, firmado, el 2 de Febrero de 1948, entre los Estados Unidos e Italia, que dice, en el inciso $2^{\circ}$ del articulo $\mathrm{V}$ :

"La propiedad de los nacionales, Sociedades y Asociaciones de cualesquiera de las Altas Partes Contratantes, no podrá ser apropiada en el territorio de la otra sin previo juicio de derecho y sin el pronto pago de una justa y efectiva compensación. El recibo de esta compensación deberá --de conformidad con las lzyes y reglamentos vigentes que no se oponen al inciso $3^{\circ}$ del artículo XVII de este Tratado - permitirse sin inte:ferencia para retirar la compensación, mediante la obtención de cambio extranjero, en la moneda de la Alta Parte Contratante, de la que el recibidor es nacional, sociedad o asociación, en las condiciones más favorables aplicables a tal moveda en el instante de la expropiación, debiendo ser exonerado de todo impuesto de transferencia o remisión, cuando se solicita tales cambios dentro del año de recibida la compensación a la que se refiere" ${ }^{43}$.

Casi idénticas disposiciones se hailan en el Tratado de Amistad, Cómercio y Navegación firmado, el 4 de Noviembre de 1946, entre los Estados Unidos y la República China ${ }^{44}$, y el Tratado de Amistad, Comercio y Navegación firmado entre los Estados Unidos e Irlanda, el 21 de Enero de $1950^{45}$.

Se puede ver fácilmente, por consiguiente, que los Estados Unidos no tienen un modelo uniforme de Tratados para la protección de las inversiones en el extranjero. Mientras, en algunos tratados, se hace un esfuerzo para garantizar una justa, adecuada y pronta compensación en la forma indicada, otros di'uyen este requerimiento hasta el punto que el pago de la compensación en caso de expropiación se convierte en algo altamente ilusorio.

En vista de esta úitima posibilidad, se han presentado sugerencias por diversas agrupaciones, entre las que deben mencionarse especialmente la Cámara Internacional de Comercio y la Sociedad Alemana para lograr la Protección de las Inversiones Extranjeras. La Sociedad Alemana propuso en 1957 un Código titulado "Convención Internacional para la Protección Mutua de los Derechos de la Propiedad Privada en Países extranjeros" "4, que está destinado a servir de base para negociaciones entre las Naciones. Tres aspectos de este Código son especialmente significativos. Frimero: el Código otorga su protección a los inversionistas privados por un período de

4 Para el texto, ver Bishop. op. cit., nota ut supra 31. p. 487.

14 Artículo VI, par 2. Para texto, ver 43 Am. J. Int. L., Supp. 27 (1949).

45 Articulo VIII, par. 2. Texto publicado por Wilson. ((Property-Protection Provision in United States Commercial Treaties" 45 Am. J. Int. L. 83, 103 (1951).

16 Para una completa discusión sobre este Código, ver Miller, "Protection of Private Foreign Investment by Multilateral Convention", 53 Am. J. Int. L. 371 (1959). 
treinta años desde la fecha de la inversión, de manera que la expropiación no podrá efectuarse durante ese plazo. Segundo: si la expropiación debe realizarse, la propiedad privada sólo puede ser apropiada por motivos de utilidad púb'ica y mediante el pronto pago de la compensación adecuada y efectiva. Contrariamente a los tratados de los Estados Unidos que hemos mencionado, el Código explica claramente lo que significa pronta, adecuada y efectiva compensación. Es asi como pronta compensación se define como el pago en el momento de la apropiación o por lo menos el depósito en ese momento para efectuar posteriormente el pago de la compensación dentro de un plazo razonable; compensación adecuada significa el precio favorable en el mercado de la propiedad expropiada; y efectiva compensación es el pago en la moneda del inversionista o en una moneda convertible a dicha moneda ". - Finalmente, una Corte Internacional se estableceria para la solución de los conflictos que podrian surgir entre las partes.

No puede dudarse que este Código constituye el instrumento que más lejos ha llegado en cuanto a la protección de las inversiones. Mientras puedan haber grandes dudas desde el punto de saber si este Código es declaratorio de principios ya existentes del derecho internacional, debe recordarse que el derecho tradicional ha sido considerado como inadecuado en esta época tan vulnerable para las vicisitudes de las conveniencias politicas. Deberia reconocerse francamente que la única manera de proteger efectivamente las inversiones es mediante una convención internacional de la más elevada autoridad, tal como lo sugiere la propuesta de la Asociación Alemana. Aún cuando esta conclusión no se halle libre de dificultades, bajo las actuales condiciones seria la solución más deseable. Se podria argüir persuasivamente, por otra parte, que los tratados biiaterales firmados por los Estados Unidos y otros paises demuestran notablemente bien el poco deseo que tienen algunos de ellos para comprometerse por adelantado al pago de compensación en la forma que sugiere el Código Alemán. A esto debe agregarse la política de los Estados Unidos de no solicitar compensación en términos tan rígidos de los paises en peligro de convulsiones sociales por temor a verlos caer dentro de la órbita comunista. Estas son consideracicnes que seguramente han guiado la politica del Gobierno de los Estados Unidos sobre el asunto y que explicaria parcialmente porque no han insistido sobre disposiciones más rigurosas hacia el pago de compensaciones en los tratados firmados con paises extranjeros.

\section{IV.-Conclusión.}

La evidencia reunida en este trabajo demuestra claramente que las ga. rantias para la protección de las inversiones están cargadas de incertidum-e bres motivadas por razones politicas. Esta conclusión, sin embargo, no afec- 
ta el deseo de establecer cierta medida de protección mediante una convención internacional. Las dificultadés que hemos subrayado están únicamente destinadas a mostrar las complejidades inherentes a esta tarea. Se puede. por lo tanto, esperar que una convención internacional que proteja la propiedad privada extranjera sea negociada, estableciendo garantias con la mayor precisión posible. Desde que el mayor bienestar de la mayor parte de la humanidad depende de la libre circulación del capital y que éste no se invertirá actualmente en países donde existen amplios programas de nacionalización, la "negociación de una especie de Converición internacional que proteja las inversiones extranjeras, es algo que no puede ser demorado en adelante.

- NOTA. - Publicado por el "Institute on Corporate Counsel", de la Ulu'versidad de Fordham (Forefgr operations of American Businees Enterprises 1961). Traducido del inglés por Javier Kiefer-Marchand $y$ publicado co nautorixación del autor, Dr. Garcia-Mora, y de la mencionada Universidad. 